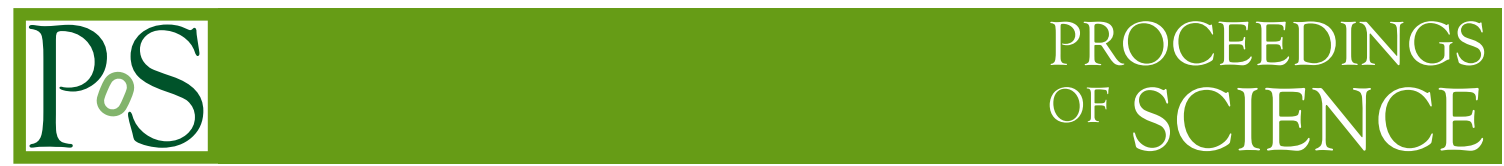

\title{
Non oscillation flavor physics at future neutrino oscillation facilities
}

\section{Osamu Yasuda*}

Department of Physics, Tokyo Metropolitan University, Hachioji, Tokyo 192-0397, Japan E-mail: yasuda AT phys.metro-u.ac.jp

New physics which can be probed at future long baseline experiments is discussed. These include the exotic interactions which modify the neutrino mixing at production, in propagation and at detection, and violation of unitarity which arises due to the effect of heavy particles.

10th International Workshop on Neutrino Factories, Super beams and Beta beams

June 30 - July 52008

Valencia, Spain

${ }^{*}$ Speaker. 


\section{Introduction}

It is expected that future long baseline neutrino experiments such as super-beams, beta-beams, neutrino factories will have great sensitivity to the third mixing angle $\theta_{13}$, the CP phase $\delta$ and the mass hierarchy $\operatorname{sign}\left(\Delta m_{31}^{2}\right)$ (For a review, see, e.g., Ref. [1]). As is the case with the B factories, great precision in the experiments will allow us to probe new physics by looking for a deviation from the standard model with neutrino mass.

A class of effective non-standard neutrino interactions with matter which will be discussed here are

$$
\mathscr{L}_{\mathrm{eff}}^{\mathrm{NSI}}=\left\{\begin{array}{l}
-2 \sqrt{2} \varepsilon_{\alpha \beta}^{\prime f P} G_{F}\left(\bar{v}_{\alpha} \gamma_{\mu} P_{L} \ell_{\beta}\right)\left(\bar{f} \gamma^{\mu} P f^{\prime}\right) \\
-2 \sqrt{2} \varepsilon_{\alpha \beta}^{f P} G_{F}\left(\bar{v}_{\alpha} \gamma_{\mu} P_{L} v_{\beta}\right)\left(\bar{f} \gamma^{\mu} P f^{\prime}\right)
\end{array}\right.
$$

Here $f$ and $f^{\prime}$ stand for fermions (the only relevant ones are electrons, $\mathrm{u}$ and d quarks), $G_{F}$ is the Fermi coupling constant, $P$ stands for a projection operator and is either $P_{L} \equiv\left(1-\gamma_{5}\right) / 2$ or $P_{R} \equiv\left(1+\gamma_{5}\right) / 2$. The interaction (1.1) is the most general form of the interactions which conserve electric charge, color, and lepton number [2]. The interactions Eq. (1.1) (a) and (b) correspond to charged and neutral current interactions, respectively. The presence of the interaction of Eq. (1.1) (a) would change the process of production and detection of neutrinos, while that of Eq. (1.1) (b) would modify the matter effect during propagation of neutrinos.

The exotic interactions (1.1) are supposed to come from some new physics beyond the standard model, but we do not specify any particular dynamics which produces (1.1) here. If these interactions come from dimension-six operators such as $\left(H^{\dagger} \bar{L}_{\alpha}\right) \gamma^{\mu} i D_{\mu}\left(H L_{\beta}\right)$, where $H$ and $L$ denote $S U(2)_{L}$ doublet of the higgs and lepton, respectively, then the coefficient of this term would be strongly constrained by charged lepton processes. In order to avoid the strong constraints on $\varepsilon_{\alpha \beta}$, therefore, we have to assume that the operator in Eq. (1.1) originates from the dimension-eight one such as $\left(\bar{L}_{\alpha} P_{R} H^{c}\right) \gamma_{\mu}\left(\left(H^{c}\right)^{\dagger} P_{L} L_{\beta}\right) \bar{f} \gamma^{\mu} P f$, which produces the operator in Eq. (1.1) (b) after the $S U(2)_{L}$ breaking. In this case the constraint on $\varepsilon_{\alpha \beta}^{f P}$ can be obtained only by the experiment with neutrinos.

\section{New Physics in oscillation experiments}

In the presence of the interaction in Eq. (1.1) (a), not only the process $\pi^{+} \rightarrow \mu^{+}+v_{\mu}$ but also $\pi^{+} \rightarrow \mu^{+}+v_{e}$ occurs with the weight $1: \varepsilon_{e \mu}^{s}$, where $s$ stands for source. These processes can be expressed as $\pi^{+} \rightarrow \mu^{+}+v_{\mu}^{s}$ where the modified flavor eigenstates $v_{\alpha}^{s}$ at source are defined by

$$
\left(\begin{array}{c}
v_{e}^{s} \\
v_{\mu}^{s} \\
v_{\tau}^{s}
\end{array}\right)=U^{s}\left(\begin{array}{c}
v_{e} \\
v_{\mu} \\
v_{\tau}
\end{array}\right), \quad U^{s} \equiv\left(\begin{array}{ccc}
1 & -\varepsilon_{e \mu}^{s} & -\varepsilon_{e \tau}^{s} \\
\varepsilon_{\mu e}^{s} & 1 & -\varepsilon_{\mu \tau}^{s} \\
\varepsilon_{\tau e}^{s} & \varepsilon_{\tau \mu}^{s} & 1
\end{array}\right)
$$

Similarly, the same phenomena happens at detection. For instance, the processes $v_{e}+n \rightarrow e^{-}+p$ and $v_{e}+n \rightarrow \mu^{-}+p$ occur with the weight $1: \varepsilon_{e \mu}^{d}$, where $d$ stands for detection. So these processes can be expressed as $v_{e}^{d}+n \rightarrow e^{-}+p$, where the modified flavor eigenstates $v_{\alpha}^{d}$ at detection are 
defined by

$$
\left(\begin{array}{c}
v_{e}^{d} \\
v_{\mu}^{d} \\
v_{\tau}^{d}
\end{array}\right)=U^{d}\left(\begin{array}{c}
v_{e} \\
v_{\mu} \\
v_{\tau}
\end{array}\right), \quad U^{d} \equiv\left(\begin{array}{ccc}
1 & -\varepsilon_{e \mu}^{d} & -\varepsilon_{e \tau}^{d} \\
\varepsilon_{\mu e}^{d} & 1 & -\varepsilon_{\mu \tau}^{d} \\
\varepsilon_{\tau e}^{d} & \varepsilon_{\tau \mu}^{d} & 1
\end{array}\right)
$$

In the presence of the new interaction of Eq. (1.1) (b), by introducing the notation $\varepsilon_{\alpha \beta}^{m} \equiv$ $\sum_{P}\left(\varepsilon_{\alpha \beta}^{e P}+3 \varepsilon_{\alpha \beta}^{u P}+3 \varepsilon_{\alpha \beta}^{d P}\right)$, and by making the approximation that the number density of electrons $\left(N_{e}\right)$, protons and neutrons are equal, the $3 \times 3$ matrix of the matter potential becomes

$$
\mathscr{A} \equiv A\left(\begin{array}{ccc}
1+\varepsilon_{e e}^{m} & \varepsilon_{e \mu}^{m} & \varepsilon_{e \tau}^{m} \\
\varepsilon_{\mu e}^{m} & \varepsilon_{\mu \mu}^{m} & \varepsilon_{\mu \tau}^{m} \\
\varepsilon_{\tau e}^{m} & \varepsilon_{* \mu}^{m} & \varepsilon_{\tau \tau}^{m}
\end{array}\right)
$$

where $A \equiv \sqrt{2} G_{F} N_{e}$.

In total, in the presence of the new interactions of Eq. (1.1) (a) and (b), the oscillation probability at length $L$ of the neutrino path can be written as

$$
\begin{aligned}
P\left(v_{\alpha} \rightarrow v_{\beta}\right)= & \left|\left[U^{d} \tilde{U} \exp (-i \tilde{\mathscr{E}} L) \tilde{U}^{\dagger} U^{s^{\dagger}}\right]_{\beta \alpha}\right|^{2} \\
= & \left|\left(U^{d} U^{s \dagger}\right)_{\beta \alpha}\right|^{2}-4 \operatorname{Re} \sum_{j<k}\left(\tilde{X}_{j}^{\beta \alpha} \tilde{X}_{k}^{\beta \alpha *}\right) \sin ^{2}\left(\frac{\Delta \tilde{E}_{j k} L}{2}\right) \\
& +2 \operatorname{Im} \sum_{j<k}\left(\tilde{X}_{j}^{\beta \alpha} \tilde{X}_{k}^{\beta \alpha *}\right) \sin \left(\Delta \tilde{E}_{j k} L\right),
\end{aligned}
$$

where $\tilde{X}_{j}^{\beta \alpha} \equiv\left(U^{d} \tilde{U}\right)_{\beta j}\left(U^{s} \tilde{U}\right)_{\alpha j}^{*}, \tilde{U}$ and $\tilde{\mathscr{E}}$ are defined by diagonalization of a $3 \times 3$ matrix in matter: $U \operatorname{diag}\left(E_{j}\right) U^{\dagger}+\mathscr{A} \equiv \tilde{U} \tilde{\mathscr{E}} \tilde{U}^{\dagger}, \tilde{\mathscr{E}} \equiv \operatorname{diag}\left(\tilde{E}_{j}\right), E_{j}$ and $\tilde{E}_{j}$ are the energy eigenvalues in vacuum and matter, respectively, and $\Delta \tilde{E}_{j k} \equiv \tilde{E}_{j}-\tilde{E}_{k}$. As in the case of the standard neutrino scenario [3, 4], the quantity $\tilde{X}_{j}^{\beta \alpha}$ can be expressed in terms of the quantity $X_{j}^{\alpha \beta} \equiv U_{\alpha j}^{*} U_{\beta j}$ in vacuum, $U^{s}, U^{d}, \mathscr{A}$ and $\tilde{E}_{j}$, i.e., $\tilde{X}_{j}^{\beta \alpha}=\sum_{\ell=1}^{3}\left(V^{-1}\right)_{j \ell}\left[U^{d}\left\{U \operatorname{diag}\left(E_{j}\right) U^{\dagger}+\mathscr{A}\right\}^{\ell-1} U^{s \dagger}\right]_{\beta \alpha}$, where $V^{-1}$ is the inverse of the Van der Monde matrix $V$, which is defined by $(V)_{j k}=\tilde{E}_{k}^{j-1}$.

\section{New Physics at source and detector [5]}

\subsection{Current bounds on $\varepsilon_{\alpha \beta}^{s, d}$ [5]}

To see the effect of New Physics at source and detector, it is advantageous to take the limit $L \rightarrow 0$ in Eq. (2.4), so that the effect of the oscillation becomes negligible. In this case we get

$$
\lim _{L \rightarrow 0} P\left(v_{\alpha} \rightarrow v_{\beta}\right)=\left|\left(U^{d} U^{s \dagger}\right)_{\beta \alpha}\right|^{2} \simeq\left|\varepsilon_{\beta \alpha}^{s}-\varepsilon_{\beta \alpha}^{d}\right|^{2} \sim \max \left(\left|\varepsilon_{\beta \alpha}^{s}\right|^{2},\left|\varepsilon_{\beta \alpha}^{d}\right|^{2}\right) .
$$

where we have assumed that there is no accidental cancellation between $\varepsilon_{\beta \alpha}^{s}$ and $\varepsilon_{\beta \alpha}^{d}$. Using Eq. (3.1), we can put bounds on the $\varepsilon_{\alpha \beta}^{s, d}$ parameters from the negative results at short baseline experiments.

It has been shown by taking into account various experimental constraints that the absolute value of the coefficient $\varepsilon_{\alpha \beta}^{f P}$ of the interaction of type Eq. (1.1) (a) is small:

$$
\left|\varepsilon_{e \mu}^{s, d}\right|<6 \times 10^{-2} \text { (MiniBooNE), }\left|\varepsilon_{\mu \tau}^{s, d}\right|<1 \times 10^{-2} \text { (NOMAD), }\left|\varepsilon_{e \tau}^{s, d}\right|<1 \times 10^{-1} \text { (NOMAD). }
$$




\subsection{Sensitivity to $\varepsilon_{\alpha \beta}^{s, d}$ in future experiments}

In general, sensitivity obtained by theorists becomes worse as times goes by, because more and more sophisticated aspects (such as systematic errors, correlation of errors, parameter degeneracy, etc.) are taken into account in the later analysis, and these factors usually give more pessimistic results than those with statistical errors only.

Sensitivity to $\varepsilon_{\alpha \beta}^{s, d}$ has been investigated by several authors. Ref. [6] gave the sensitivity $\left|\varepsilon_{e \mu}^{s}\right|<$ a few $\times 10^{-4}$ at a neutrino factory. Ref. [7] obtained the sensitivity at a neutrino factory: $\left|\varepsilon_{e \mu}^{s}\right|<$ $3 \times 10^{-3},\left|\varepsilon_{e \tau}^{s}\right|<3 \times 10^{-3}$ from $v_{e} \rightarrow v_{\mu}$ and $\left|\varepsilon_{\mu \tau}^{s}\right|<3 \times 10^{-3}$ from $v_{\mu} \rightarrow v_{\tau}$. Ref. [8] obtained the sensitivity $\left|\varepsilon_{e \mu}^{s}\right|<3 \times 10^{-2}$ by combining T2K and Double CHOOZ, and $\left|\varepsilon_{e \mu}^{s}\right|<1.5 \times 10^{-2}$ by combining Nova and a DCHOOZ-like 200kt reactor experiment.

\section{New Physics in propagation (matter effect) $[9,10]$}

\subsection{Constraints from various neutrino experiments}

Constraints on $\varepsilon_{\alpha \beta}^{m}$ from various neutrino experiments have been discussed in Refs. [2, 11, 12, 13]. The bounds on $\varepsilon_{\alpha \beta}^{\ell P}$, which are obtained by CHARM, LEP, LSND and NuTeV as of 2003, is given in Tables 2 and 3 in Ref. [2], and the updated bounds [14] which have been improved since 2003 are given in Table 1 .

On the other hand, it was shown in Ref. [16] that the atmospheric neutrino and K2K data imply

$$
\left|\varepsilon_{e \tau}^{m}\right|^{2} \simeq \varepsilon_{\tau \tau}^{m}\left(1+\varepsilon_{e e}^{m}\right),
$$

and $\left|\varepsilon_{e \tau}^{m}\right| \lesssim\left|1+\varepsilon_{e e}^{m}\right|$. The effect of new physics in propagation to solar neutrinos is also discussed [17], but no new constraint is obtained from the results in Ref. [17].

Since the coefficients $\varepsilon_{\alpha \beta}^{m}$ in Eq. (2.3) are give by $\varepsilon_{\alpha \beta}^{m} \sim \varepsilon_{\alpha \beta}^{e}+3 \varepsilon_{\alpha \beta}^{u}+3 \varepsilon_{\alpha \beta}^{d}$, taking into account the constraints by Refs. [2] (with the update in Table 1) and [16], we have the following constraints: ${ }^{1}$

$$
\left(\begin{array}{ccc}
-4<\varepsilon_{e e}^{m}<2.6 & \left|\varepsilon_{e \mu}^{m}\right|<1.4 \times 10^{-4} & \left|\varepsilon_{e \tau}^{m}\right|<1.2 \\
& -0.05<\varepsilon_{\mu \mu}^{m}<0.08 & \left|\varepsilon_{\mu \tau}^{m}\right|<0.25 \\
& & \left|\varepsilon_{\tau \tau}^{m}\right|<19
\end{array}\right) .
$$

Notice that the only bounds on $\varepsilon_{\alpha \beta}^{m}$ which are improved by the update in Table 1 are those on $\varepsilon_{e \mu}^{m}$ and $\varepsilon_{e \tau}^{m}$ (cf. Eq. (3) in Ref. [18]).

\subsection{Phenomenology with $\varepsilon_{e e}^{m}, \varepsilon_{e \tau}^{m}, \varepsilon_{\tau \tau}^{m} \sim \mathscr{O}(1)$}

Because the present experimental constraints allow the possibility $\varepsilon_{e e}^{m}, \varepsilon_{e \tau}^{m}, \varepsilon_{\tau \tau}^{m} \sim \mathscr{O}(1)$, one could discuss the phenomenological consequences of a scenario with $\varepsilon_{\alpha \beta}^{m} \sim \mathscr{O}(1)(\alpha, \beta=e, \tau) .^{2}$

\footnotetext{
${ }^{1}$ In deriving Eq. (4.2), we have to remember that the bound of one particular parameter $\varepsilon_{\alpha \beta}^{f P}$ was obtained in Refs. [2] by assuming that all the parameters other than $\varepsilon_{\alpha \beta}^{f P}$ are zero. In the case of the bound on $\varepsilon_{e e}^{m}$, for instance, we have $\varepsilon_{e e}^{m}<\max _{P=L, R}\left\{\min _{\text {expts }}\left(\varepsilon_{e e}^{e P \text {,expts }}\right)\right\}+3 \times \max _{f=u, d ; P=L, R}\left\{\min _{\text {expts }}\left(\varepsilon_{e e}^{f P \text {,expts }}\right)\right\}=0.5+3 \times 0.7=2.6$, where we have minimized (i.e., chosen the best bound for) $\varepsilon_{\alpha \beta}^{f P \text {, expts }}$ with respect to various experimental data for fixed $\{f, P, \alpha, \beta\}$ and have maximized (chosen the weakest bound) among different $P$ for $f=e$ or $\{f, P\}$ for $f=u, d$.

${ }^{2}$ The scenario with $\varepsilon_{e e}^{m}, \varepsilon_{e \tau}^{m}, \varepsilon_{\tau \tau}^{m} \sim \mathscr{O}(1)$ may have a theoretical problem because $\varepsilon_{\alpha \beta}^{m}$ is supposed to be of order $\left(M_{W} / \Lambda_{N P}\right)^{k}(k \geq 2)$, where $\Lambda_{N P}$ stands for the scale of the new physics and and it is expected to be larger than $M_{W}$.
} 


\begin{tabular}{||c|c|c||}
\hline \hline vertex & limits in 2003 [2] & current limits [14] \\
\hline \hline$\left(\bar{e} \gamma^{\rho} P e\right)\left(\bar{v}_{\tau} \gamma_{\rho} L v_{\mu}\right)$ & $\left|\varepsilon_{\tau \mu}^{e P}\right|<1.2$ & $\left|\varepsilon_{\tau \mu}^{e P}\right|<0.15$ \\
& $(\tau \rightarrow \mu \bar{e} e) *$ & $(\tau \rightarrow \mu \bar{e} e) *$ \\
\hline$\left(\bar{u} \gamma^{\rho} P u\right)\left(\bar{v}_{\tau} \gamma_{\rho} L v_{\mu}\right)$ & $\left|\varepsilon_{\tau \mu}^{u P}\right|<2.8$ & $\left|\varepsilon_{\tau \mu}^{u P}\right|<0.29$ \\
& $(\tau \rightarrow \mu \rho) *$ & $(\tau \rightarrow \mu \rho) *$ \\
$\left(\bar{d} \gamma^{\rho} P d\right)\left(\bar{v}_{\tau} \gamma_{\rho} L v_{\mu}\right)$ & $\left|\varepsilon_{\tau \mu}^{d P}\right|<2.8$ & $\left|\varepsilon_{\tau \mu}^{d P}\right|<0.29$ \\
& $(\tau \rightarrow \mu \rho) *$ & $(\tau \rightarrow \mu \rho) *$ \\
\hline$\left(\bar{u} \gamma^{\rho} P u\right)\left(\bar{v}_{\mu} \gamma_{\rho} L v_{e}\right)$ & $\left|\varepsilon_{\mu e}^{u P}\right|<7.7 \times 10^{-4}$ & $\left|\varepsilon_{\mu e}^{u P}\right|<3.1 \times 10^{-4}$ \\
& $(\mu \mathrm{Ti} \rightarrow e \mathrm{Ti}) *$ & $(\mu \mathrm{Au} \rightarrow e \mathrm{Au}) *$ \\
\hline$\left(\bar{d} \gamma^{\rho} P d\right)\left(\bar{v}_{\mu} \gamma_{\rho} L v_{e}\right)$ & $\left|\varepsilon_{\mu e}^{d P}\right|<7.7 \times 10^{-4}$ & $\left|\varepsilon_{\mu e}^{d P}\right|<3.1 \times 10^{-4}$ \\
& $(\mu \mathrm{Ti} \rightarrow e \mathrm{Ti}) *$ & $(\mu \mathrm{Au} \rightarrow e \mathrm{Au}) *$ \\
\hline$\left(\bar{e} \gamma^{\rho} P e\right)\left(\bar{v}_{\tau} \gamma_{\rho} L v_{e}\right)$ & $\left|\varepsilon_{\tau e}^{e P}\right|<2.9$ & $\left|\varepsilon_{\tau e}^{e P}\right|<0.32$ \\
& $(\tau \rightarrow e \bar{e} e) *$ & $(\tau \rightarrow e \bar{e} e) *$ \\
\hline$\left(\bar{u} \gamma^{\rho} P u\right)\left(\bar{v}_{\tau} \gamma_{\rho} L v_{e}\right)$ & $\left|\varepsilon_{\tau e}^{u P}\right|<1.6$ & $\left|\varepsilon_{\tau e}^{u P}\right|<0.28$ \\
& $(\tau \rightarrow e \rho) *$ & $(\tau \rightarrow e \rho) *$ \\
\hline$\left(\bar{d} \gamma^{\rho} P d\right)\left(\bar{v}_{\tau} \gamma_{\rho} L v_{e}\right)$ & $\left|\varepsilon_{\tau e}^{d P}\right|<1.6$ & $\left|\varepsilon_{\tau e}^{d P}\right|<0.28$ \\
& $(\tau \rightarrow e \rho) *$ & $(\tau \rightarrow e \rho) *$ \\
\hline \hline
\end{tabular}

Table 1: The updated bounds in Table 3 in [2] (see Ref. [2] for details). Some of the bounds have been updated even further using the data in Ref. [15] after the talk. (Courtesy of Sacha Davidson)

In this case it is known [18] that the appearance channel $v_{\mu} \rightarrow v_{e}$ gets enhanced for relatively long baseline lengths $\gtrsim 1000 \mathrm{~km}$, and if $\varepsilon_{e \tau}^{m}$ is very large within the current bound then MINOS may be able to show the existence of new physics from $v_{e}$ appearance [19, 20, 21]. In such an analysis, it is useful to have the analytic expression for the oscillation probability $P\left(v_{\mu} \rightarrow v_{e}\right)$, and it was obtained in Ref. [22] using the method by Kimura, Takamura and Yokomakura [3, 4].

It was pointed out in Ref. [23] that the disappearance channel $v_{\mu} \rightarrow v_{\mu}$ could also play a role to determine the parameters from the relations $\sin ^{2} 2 \theta_{\mathrm{atm}} / \sin ^{2} 2 \theta_{23}=\left(1+c_{\beta}^{2}\right)^{2} / 4 c_{\beta}^{2},\left|\Delta m_{\mathrm{atm}}^{2} / \Delta m_{23}^{2}\right|=$ $2 c_{\beta}^{2} /\left(1+c_{\beta}^{2}\right)$, where $\beta$ is defined through $\tan \beta=\left|\varepsilon_{e \tau}^{m}\right| /\left(1+\varepsilon_{e e}^{m}\right)$, and $\sin ^{2} 2 \theta_{\text {atm }}$ and $\left|\Delta m_{\text {atm }}^{2}\right|$ are the values determined by the atmospheric neutrino experiments which observe potential matter effects due to the new physics. In the near future, T2K, which has the baseline length $295 \mathrm{~km}$ and therefore suffers little from the matter effects, is expected to measure $\sin ^{2} 2 \theta_{23}$ and $\left|\Delta m_{31}^{2}\right|$ precisely. If the central value of these two quantities by T2K turn out to be very close to those by the atmospheric neutrino data, however, the errors in the atmospheric neutrino oscillation parameters [24] $\sin ^{2} 2 \theta_{\text {atm }}(+0 \%-6 \%)$ and $\left|\Delta m_{\mathrm{atm}}^{2}\right|( \pm 20 \%)$ will remain dominant, so the bound on $\left|\varepsilon_{e \tau}^{m}\right|$ is unfortunately not expected to improve very much.

\subsection{Sensitivity to $\varepsilon_{\alpha \beta}^{m}$ in future experiments}

Since the matter effect appears in the oscillation probability at distance $L$ in the form of $A L \equiv$ $\sqrt{2} G_{F} N_{e} L \sim L /(2000 \mathrm{~km})$, in order to measure $\varepsilon_{\alpha \beta}^{m}$ precisely, it is necessary for the baseline length $L$ to be larger than $\mathscr{O}(1000 \mathrm{~km})$, and neutrino factories are ideal for that purpose. 
Ref. [25] gave the sensitivity at a neutrino factory $\left|\varepsilon_{\tau \tau}^{m}\right|<$ several $\times 10^{-1}$ and $\left|\varepsilon_{\mu \tau}^{m}\right|<$ a few $\times$ $10^{-3}$ from $v_{\mu} \rightarrow v_{\tau}$ where $\varepsilon_{e e}^{m}=\varepsilon_{e \tau}^{m}=0$ is assumed, and $\left|\varepsilon_{e \tau}^{m}\right|<$ several $\times 10^{-3}$ from $v_{e} \rightarrow v_{\mu}$ where $\varepsilon_{\alpha \mu}^{m}=0$ is assumed. Ref. [26] pointed out that degeneracy between $\theta_{13}$ and $\varepsilon_{e \tau}^{m}$ can be resolved by considering the $v_{e} \rightarrow v_{\mu}$ channel at a neutrino factory at baselines $3000 \mathrm{~km}$ and $7000 \mathrm{~km}$. Using the $v_{e} \rightarrow v_{\tau}$ channel, Ref. [27] found the sensitivity $\left|\varepsilon_{e \tau}^{m}\right|<1 \times 10^{-2}$. Ref. [2] discussed the potential at near detectors of a neutrino factory using leptonic $\sin ^{2} \theta_{W}$ and $\sin ^{2} \theta_{W}$ in DIS, and gave the sensitivity $(f=e, u, d)$ : $\left|\varepsilon_{\alpha \alpha}^{f}\right|<1 \times 10^{-3}(\alpha=e, \mu),\left|\varepsilon_{\alpha \tau}^{f}\right|<1 \times 10^{-3}(\alpha=e, \mu)$. Assuming $\varepsilon_{\alpha \mu}^{m}=0(\alpha=e, \mu, \tau)$, Ref. [28] gave the bound at a neutrino factory $\left|\varepsilon_{e \tau}^{m}\right|<$ a few $\times 10^{-3}$. Ref. [8] gave the bound not only on $\varepsilon_{\alpha \beta}^{s, d}$, but also on $\varepsilon_{\alpha \beta}^{m}$ by combination of the accelerator and reactor experiments. They obtained $\left|\varepsilon_{e \mu}^{m}\right|<0.5$ from T2K and Double CHOOZ, and $\left|\varepsilon_{e \mu}^{m}\right|<0.1$ from Nova and a DCHOOZ-like 200kt reactor experiment. In the two flavor framework with $v_{\mu}$ and $v_{\tau}$ (i.e., by assuming $\left.\varepsilon_{e \alpha}^{m}=0(\alpha=e, \mu, \tau)\right)$, Ref. [29] gave the bound $\left|\varepsilon_{\mu \tau}^{m}\right|<0.03$ and $\left|\varepsilon_{\tau \tau}^{m}\right|<0.3$ at T2KK. Ref. [30] examined if OPERA helps to resolve $\theta_{13}-\varepsilon_{e \tau}^{m}$ degeneracy, but unfortunately statistics at OPERA turned out to be too small to be significant to constrain $\varepsilon_{e \tau}^{m}$. Ref. [31] obtained sensitivity to various parameters at a neutrino factory. $\left|\varepsilon_{e e}^{m}\right| \lesssim 0.1,\left|\varepsilon_{e \tau}^{m}\right| \lesssim$ several $\times 10^{-3},\left|\varepsilon_{\mu \tau}^{m}\right| \lesssim$ a few $\times 10^{-2}$, $\left|\varepsilon_{\tau \tau}^{m}\right| \lesssim$ a few $\times 10^{-2}$. Ref. [32] discussed sensitivity to $\operatorname{Re}\left(\varepsilon_{\mu \tau}^{m}\right)$ and $\operatorname{Im}\left(\varepsilon_{\mu \tau}^{m}\right)$ at OPERA.

\section{Violation of unitarity}

It was pointed out in Ref. [33] that in generic see-saw models the kinetic term gets modified after integrating out the right handed neutrino and unitarity is expected to be violated. ${ }^{3}$ When the mixing matrix $N$ is nonunitary, it is in general written as $N=H U$ where $U$ is unitary and $H$ is hermitian. Deviation from unitarity is expressed as $N N^{\dagger}-1$, and because deviation from unitarity is expected to be small, $N N^{\dagger}=H^{2}$ is close to identity. In the case of the socalled minimal unitarity violation, in which only three light neutrinos are involved and sources of unitarity violation are assumed to appear only in the neutrino sector, $N N^{\dagger}-1$ have strong constraints, which mostly comes from the constraints of rare decays of charged leptons, and its matrix elements are smaller than $\mathscr{O}(1 \%)$ [33]. In practice the modified oscillation probability $\hat{P}\left(v_{\alpha} \rightarrow v_{\beta}\right) \equiv P\left(v_{\alpha} \rightarrow v_{\beta}\right)\left(N N^{\dagger}\right)_{\alpha \alpha}\left(N N^{\dagger}\right)_{\beta \beta}$ turns out to be useful, and it is given by [34]

$$
\begin{aligned}
\hat{P}\left(v_{\alpha} \rightarrow v_{\beta}\right)= & \left|\left(N^{*} N^{T}\right)_{\alpha \beta}\right|^{2}-4 \sum_{j<k} \operatorname{Re}\left(\tilde{\mathscr{X}}_{j}^{\alpha \beta} \tilde{\mathscr{X}}_{k}^{\alpha \beta *}\right) \sin ^{2}\left(\frac{\Delta \tilde{E}_{j k} L}{2}\right) \\
& +2 \sum_{j<k} \operatorname{Im}\left(\tilde{\mathscr{X}}_{j}^{\alpha \beta} \tilde{\mathscr{X}}_{k}^{\alpha \beta *}\right) \sin \left(\Delta \tilde{E}_{j k} L\right),
\end{aligned}
$$

where $\tilde{\mathscr{X}}_{j}^{\alpha \beta} \equiv\left(N^{*} W\right)_{\alpha j}\left(N W^{*}\right)_{\beta j}(j=1,2,3), W$ is a unitary matrix which diagonalizes the hermitian energy matrix: $\mathscr{E}+N^{T} \mathscr{A} N^{*}=W \tilde{\mathscr{E}} W^{-1}, \tilde{\mathscr{E}}=\operatorname{diag}\left(\tilde{E}_{j}\right), \tilde{E}_{j}$ is the energy eigenvalue, and $\Delta \tilde{E}_{j k} \equiv \tilde{E}_{j}-\tilde{E}_{k} . \tilde{X}_{j}^{\alpha \beta}$ can be expressed in terms of the quantity $X_{j}^{\alpha \beta} \equiv U_{\alpha j}^{*} U_{\beta j}$ in vacuum, $H$, $\mathscr{A}$ and $\tilde{E}_{j}$, i.e., $\tilde{X}_{j}^{\beta \alpha}=\sum_{\ell=1}^{3}\left(V^{-1}\right)_{j \ell}\left[N^{*}\left\{\operatorname{diag}\left(E_{j}\right)+N^{T} \mathscr{A} N^{*}\right\}^{\ell-1} N^{T}\right]_{\beta \alpha}$, where $V^{-1}$ is again the inverse of the Van der Monde matrix $(V)_{j k}=\tilde{E}_{k}^{j-1}$.

\footnotetext{
${ }^{3}$ The nontrivial issue is the magnitude of violation. Some of see-saw models (e.g., inverse see-saw) do have two scales, one to produce small neutrino mass and another which may not be extremely different from $M_{W}$. Then magnitude of violation may not be extremely small.
} 
Sensitivity to violation of unitarity was examined in Ref. [33] by assuming a neutrino factory with a 4kt OPERA-like near detector at 100m and they got the bound $\left|\left(N N^{\dagger}\right)_{e \tau}\right|<2.9 \times 10^{-3}$ from $v_{e} \rightarrow v_{\tau}$ (cf. the current bound 0.016) and $\left|\left(N N^{\dagger}\right)_{\mu \tau}\right|<2.6 \times 10^{-3}$ from $v_{\mu} \rightarrow v_{\tau}$ (cf. the current bound 0.013). As in the case of new physics at production and detection, experiments at shorter baselines are expected to be advantageous to observe violation of unitarity, and Ref. [34] studied sensitivity to $(H-\mathbf{1})_{\mu \tau}$ at a neutrino factory with a $5 \mathrm{kt}$ OPERA-like far detector at $130 \mathrm{~km}$, where $H$ is the hermitian matrix which appears in the decomposition $N=H U$, and the bound is $\left|(H-\mathbf{1})_{\mu \tau}\right|<$ several $\times 10^{-4}$ for some region of $\arg \left[(H-\mathbf{1})_{\mu \tau}\right]$.

\section{Summary}

Current bounds on the parameters $\varepsilon_{\alpha \beta}^{s, d}$, which describe new physics effects at production or detection of neutrinos, are typically of order $10^{-3} \cdot \varepsilon_{\alpha \beta}^{m}$, which describe new physics effects during propagation, have bounds typically of order $10^{-2}$, but presently the three parameters $\varepsilon_{e e}^{m}, \varepsilon_{e \tau}^{m}, \varepsilon_{\tau \tau}^{m}$ are still allowed to be of $\mathscr{O}(1)$. Neutrino factories may be able to improve bounds on $\varepsilon_{\alpha \beta}^{m}$ dramatically. Deviation from unitarity is expected in generic models (e.g., see-saw), but phenomenologically its magnitude is less than $\mathscr{O}(1 \%)$.

There are a lot of problems to be worked out on new physics which can be probed at future long baseline experiments. Some of the problems are: resolution of correlations of errors, degeneracies, etc. in the presence of all new physics parameters $\varepsilon_{\alpha \beta}^{s, m, d}$; distinction between the new physics effects (e.g., 4-fermi interactions vs. unitarity violation due to modification in the kinetic term), etc. Further studies are necessary to exhaust all possible physics at future long baseline experiments.

\section{Acknowledgments}

The author would like to thank the organizers for invitation, Sacha Davidson for providing me the updates for Table 3 in Ref. [2], and Hiroaki Sugiyama for discussions and communications, which were useful in preparing this talk, during the collaboration on [18]. Research of the author was supported in part by the JSPS Bilateral Joint Projects (Japan-Spain) and a Grant-in-Aid for Scientific Research of the Ministry of Education, Science and Culture, \#19340062.

\section{References}

[1] A. Bandyopadhyay et al. [ISS Physics Working Group], arXiv:0710.4947 [hep-ph].

[2] S. Davidson, C. Pena-Garay, N. Rius and A. Santamaria, JHEP 0303 (2003) 011 [arXiv:hep-ph/0302093].

[3] K. Kimura, A. Takamura and H. Yokomakura, Phys. Lett. B 537 (2002) 86 [arXiv:hep-ph/0203099].

[4] K. Kimura, A. Takamura and H. Yokomakura, Phys. Rev. D 66 (2002) 073005 [arXiv:hep-ph/0205295].

[5] Y. Grossman, Phys. Lett. B 359 (1995) 141 [arXiv:hep-ph/9507344].

[6] M. C. Gonzalez-Garcia, Y. Grossman, A. Gusso and Y. Nir, Phys. Rev. D 64 (2001) 096006 [arXiv:hep-ph/0105159]. 
[7] T. Ota, J. Sato and N. a. Yamashita, Phys. Rev. D 65 (2002) 093015 [arXiv:hep-ph/0112329].

[8] J. Kopp, M. Lindner, T. Ota and J. Sato, Phys. Rev. D 77 (2008) 013007 [arXiv:0708.0152 [hep-ph]].

[9] M. M. Guzzo, A. Masiero and S. T. Petcov, Phys. Lett. B 260 (1991) 154.

[10] E. Roulet, Phys. Rev. D 44 (1991) 935.

[11] Z. Berezhiani and A. Rossi, Phys. Lett. B 535 (2002) 207 [arXiv:hep-ph/0111137].

[12] J. Barranco, O. G. Miranda, C. A. Moura and J. W. F. Valle, Phys. Rev. D 73 (2006) 113001 [arXiv:hep-ph/0512195].

[13] J. Barranco, O. G. Miranda, C. A. Moura and J. W. F. Valle, Phys. Rev. D 77 (2008) 093014 [arXiv:0711.0698 [hep-ph]].

[14] S. Davidson, private communications (2008).

[15] C. Amsler et al. [Particle Data Group], Phys. Lett. B 667 (2008) 1.

[16] A. Friedland and C. Lunardini, Phys. Rev. D 72 (2005) 053009 [arXiv:hep-ph/0506143].

[17] A. Friedland, C. Lunardini and C. Pena-Garay, Phys. Lett. B 594 (2004) 347 [arXiv:hep-ph/0402266].

[18] N. Kitazawa, H. Sugiyama and O. Yasuda, arXiv:hep-ph/0606013.

[19] H. Sugiyama, AIP Conf. Proc. 981 (2008) 216 [arXiv:0711.4303 [hep-ph]].

[20] O. Yasuda, Acta Phys. Polon. B 38 (2007) 3381 [arXiv:0710.2601 [hep-ph]].

[21] M. Blennow, T. Ohlsson and J. Skrotzki, Phys. Lett. B 660 (2008) 522 [arXiv:hep-ph/0702059].

[22] O. Yasuda, arXiv:0704.1531 [hep-ph].

[23] A. Friedland and C. Lunardini, Phys. Rev. D 74 (2006) 033012 [arXiv:hep-ph/0606101].

[24] J. Raaf, talk at the 23rd International Conference on Neutrino Physics and Astrophysics (Neutrino 08), Christchurch, New Zealand, May 25-31, 2008,

http://www2 . phys. canterbury.ac.nz/ jaa53/presentations/Raaf.pdf.

[25] A. M. Gago, M. M. Guzzo, H. Nunokawa, W. J. C. Teves and R. Zukanovich Funchal, Phys. Rev. D 64 (2001) 073003 [arXiv:hep-ph/0105196].

[26] P. Huber, T. Schwetz and J. W. F. Valle, Phys. Rev. Lett. 88 (2002) 101804 [arXiv:hep-ph/0111224].

[27] M. Campanelli and A. Romanino, Phys. Rev. D 66 (2002) 113001 [arXiv:hep-ph/0207350].

[28] N. C. Ribeiro, H. Minakata, H. Nunokawa, S. Uchinami and R. Zukanovich-Funchal, JHEP 0712 (2007) 002 [arXiv:0709.1980 [hep-ph]].

[29] N. C. Ribeiro, H. Nunokawa, T. Kajita, S. Nakayama, P. Ko and H. Minakata, Phys. Rev. D 77 (2008) 073007 [arXiv:0712.4314 [hep-ph]].

[30] A. Esteban-Pretel, J. W. F. Valle and P. Huber, Phys. Lett. B 668 (2008) 197 [arXiv:0803.1790 [hep-ph]].

[31] J. Kopp, T. Ota and W. Winter, Phys. Rev. D 78 (2008) 053007 [arXiv:0804.2261 [hep-ph]].

[32] M. Blennow, D. Meloni, T. Ohlsson, F. Terranova and M. Westerberg, Eur. Phys. J. C 56 (2008) 529 [arXiv:0804.2744 [hep-ph]].

[33] S. Antusch, C. Biggio, E. Fernandez-Martinez, M. B. Gavela and J. Lopez-Pavon, JHEP 0610 (2006) 084 [arXiv:hep-ph/0607020].

[34] E. Fernandez-Martinez, M. B. Gavela, J. Lopez-Pavon and O. Yasuda, Phys. Lett. B 649 (2007) 427 [arXiv:hep-ph/0703098]. 